\section{A Typical Carcinoid Tumor of the Lung Presenting as Diffuse Cystic Bronchiectasis}

Sir,

Pulmonary carcinoid tumor is an unusual type of neuroendocrine neoplasm of lung, derived from kulchitsky cells in the basal layer of bronchial epithelium and accounts for $1-2 \%$ of all primary lung tumors. They are generally divided into typical and atypical types and considered as low-grade malignancy. ${ }^{1,2}$ The ratio between typical carcinoid (TC) and atypical carcinoid (AC) being 8-10:1.3,4 Many of carcinoids of the lung are centrally located.2 Central tumors are most often associated with signs of obstruction on computed tomography (CT) such as atelectasis, air trapping or obstructive pneumonitis, and rarely as bronchiectasis. Diffuse cystic bronchiectasis of the whole lung, secondary to a typical carcinoid tumor of lung, has not been reported before. Herein, we present a case of diffuse cystic bronchiectasis of the whole left lung in a patient who presented with recurrent pneumonia as the clinical manifestation.

A 24-year woman was admitted in this Department for recurrent pneumonia. Her problem started 2 years ago when she developed recurring cough, expectoration and hemoptysis during the late trimester of pregnancy. No special treatment was given because of pregnancy. The patient reported improvement of signs after the end of pregnancy. But, during the next 2 years, coughing and expectoration occurred when the weather got cold, with occasional hemoptysis, accompanied by fever. Chest $X$-ray showed a spot in the left lower pulmonary lobe. No other lesions in other pulmonary lobe were found. Blood tests showed an increase in white blood cell and neutrophile count, and a mild increase in other inflammatory markers. Although she received treatment for respiratory infection every time, no further examination was carried out at that time. The above symptoms could be alleviated except this time. Her symptoms persisted despite two weeks of antibiotic therapy. Because of this clinical history, on her admission to our hospital, a highresolution computed tomography (HRCT) scan of the chest was performed, which showed stenosis of left main bronchus, multiple small cystic, and tubular translucent shadows of varying size, diffusely distributed in the entire left lung, and multiple lung consolidation shadows (Figures 1 and 2). Enhanced chest CT showed more or less similar findings as on HRCT. The patient's previous physical examination was normal. There was no smoking and drinking history. There was history of weight loss (about $10 \mathrm{~kg}$ ) in the last 2 years. There was no noticeable improvement after one week of antibiotic therapy. A bronchoscopic examination followed. A nodule was seen in the lower left main bronchus at division into lobar bronchus (Figure 3). The nodule had a smooth surface. The airway was almost completely occluded. The nodule was completely excised using a high-frequency snare. Histopathology revealed a neuroendocrine tumor. Immunostaining showed the positivity for CD56, chromogranin $\mathrm{A}$, and synaptophysin, and there were no mitoses per $2 \mathrm{~mm}^{2}$ (10 HPF), which were consistent with a typical carcinoid. The patient had a successful left pneumonectomy and pathology of the resected specimen suggesting a typical stage pT2NOMO carcinoid tumor. The patient was discharged 9 days after surgery and had no recurrence at 10 months after surgery; and long-term follow-up continued.

Pulmonary carcinoid tumors on chest radiographs mostly show a well-defined hilar or peripheral mass; approximately $60 \%-80 \%$ of them are located in the central airways and about $16 \%-40 \%$ occur in the peripheral lung. 2,5 The tumors usually appear as smooth, spherical, ovoid or slightly lobulated nodules on CT, and about $30 \%$ of cases show calcification or ossification. 6 In this case, the patient was admitted in this Department for repeated respiratory tract infections accompanied by

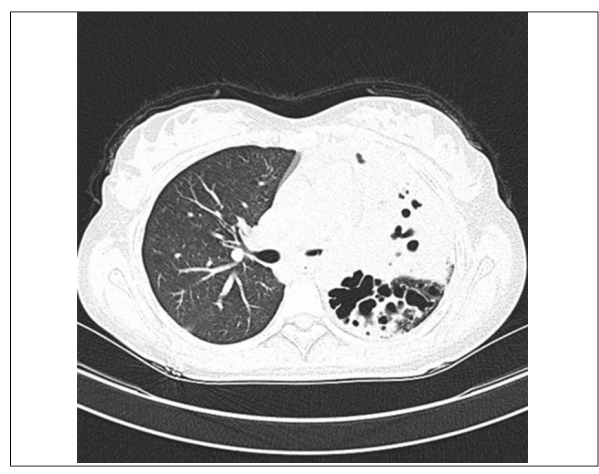

Figure 1: CT scan showing diffuse bronchiectasis of the left lung.

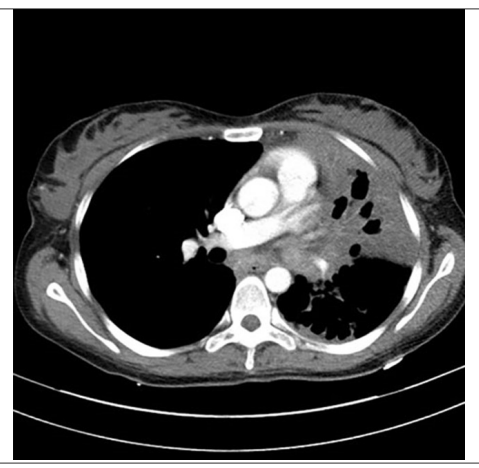

Figure 2: CT scan showing a partially obstructing lesion causing obstruction to the left lung

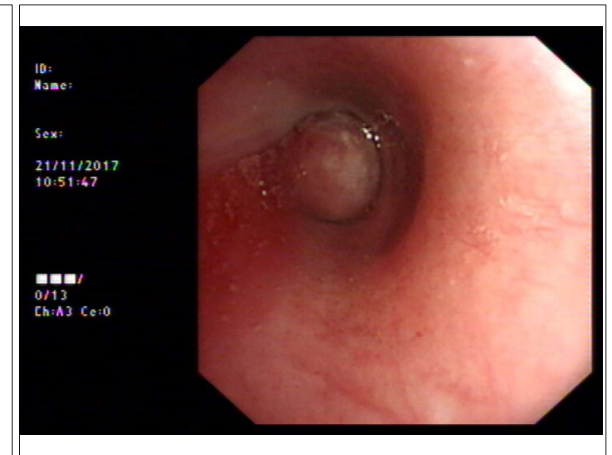

Figure 3: Bronchoscopic examination showing a nodule in the lower lobe branch of the left main bronchus. 
diffuse cystic bronchiectasis of the whole left lung on HRCT. This is the first report of a typical carcinoid tumor of the lung accompanied by diffuse cystic bronchiectasis of the whole left lung. Bronchiectasis associated with primary neoplasms is rare. Carcinoids tumors tend to grow very slowly when compared with other types of lung cancer. They, therefore, typically present with recurrent pneumonia. The persistence of inflammatory factors is an important cause of bronchiectasis. Recurrent pneumonia should be investigated by detailed examination, because this may be the sole manifestation of a potentially serious lesion. In this case, the patient's previous physical examination was normal, with no history of organic disease. She developed recurrent cough, expectoration and hemoptysis 2 years ago. If she had been investigated thoroughly at the time of first presentation, she could have been treated and the serious complication of diffuse cystic bronchiectasis could have been avoided, which ultimately required pneumonectomy.

In conclusion, physicians should keep in mind obstructing lesions when encountered with a patient with recurrent pneumonia. Despite rarity, physicians should also consider carcinoid tumors in the differential diagnosis in such cases.

\section{CONFLICT OF INTEREST:}

Authors declared no conflict of interest.

\section{AUTHORS' CONTRIBUTION:}

ZM: Wrote the manuscript.

HL: Provided the guidance.

\section{REFERENCES}

1. Hendifar AE, Marchevsky AM, Tuli R. Neuroendocrine tumors of the lung: Current challenges and advances in the diagnosis and management of well-differentiated disease. $J$ Thoracic Oncol 2017; 12:425-36.

2. Caplin ME, Baudin E, Ferolla P. Pulmonary neuroendocrine (carcinoid) tumors: European neuroendocrine tumor society expert consensus and recommendations for best practice for typical and atypical pulmonary carcinoids. Ann Oncol 2015; 26:1604-20

3. Rugge M, Fassan M, Clemente R. Bronchopulmonary carcinoid: Phenotype and long-term outcome in a single-institution series of Italian patients. Clin Cancer Res 2008; 14: 149-54.

4. Gustafsson BI, Kidd M, Chan A. Bronchopulmonary neuroendocrine tumors. Cancer 2008; 113:5-21.

5. Reuling EMBP, Dickhoff C, Plaisier PW. Endobronchial treatment for bronchial carcinoid: Patient selection and predictors of outcome. Respiration 2018; 95:220-7.

6. Chong S, Lee KS, Chung MJ. Neuroendocrine tumors of the lung: Clinical, pathologic, and imaging findings. Radiographics 2006; 26:41-57.

Zhu Meng and Huang Linian

Department of Respiration, First Affiliated Hospital of Bengbu Medical College, Anhui, China

Correspondence to: Huang Linian, Department of Respiration, First Affiliated Hospital of Bengbu Medical College, Anhui, China

E-mail:bbmchln@126.com

Received: December 14, 2018; Revised: May 20, 2019; Accepted: May 29, 2019 